\title{
Design of Maintenance Training of some Equipment Hydraulic System in Semi-physical Simulation
}

\author{
Weian Gao ${ }^{1, a}$, Xiaohu Chen ${ }^{1,}$ a and Chunjiang Yao ${ }^{1, a}$ \\ ${ }^{1}$ High Tech Research Institute, Xian 710025, China; \\ agaoweianan@163.com,
}

Keywords: hydraulic system, Kingview,PLC, maintenance training.

\begin{abstract}
This template explains and demonstrates how to prepare your camera-ready paper for Trans Tech Publications. The best is to read these instructions and follow the outline of this text. Please make the page settings of your word processor to A4 format ( $21 \times 29,7 \mathrm{~cm}$ or $8 \times 11$ inches); with the margins: bottom $1.5 \mathrm{~cm}(0.59 \mathrm{in})$ and top $2.5 \mathrm{~cm}(0.98 \mathrm{in}), \mathrm{right} /$ left margins must be $2 \mathrm{~cm}$ (0.78 in). This template explains and demonstrates how to prepare your camera-ready paper for Trans Tech Publications. The best is to read these instructions and follow the outline of this text. Please make the page settings of your word processor to A4 format $(21 \times 29,7 \mathrm{~cm}$ or $8 \times 11$ inches $)$; with the margins: bottom $1.5 \mathrm{~cm}(0.59 \mathrm{in})$ and top $2.5 \mathrm{~cm}(0.98 \mathrm{in})$, right $/$ left margins must be $2 \mathrm{~cm}(0.78 \mathrm{in})$.
\end{abstract}

\section{Introduction}

In recent years, with the deepening development of intelligent weapons and equipment, the function of the hydraulic system of the equipment is more powerful,the difficulty of the fault diagnosis and maintenance of the hydraulic system is also increasing. The way of fault diagnosis and maintenance has become increasingly unable to meet the needs of the use and maintenance of modern equipment. Development of the application of the maintenance training system for the relevant personnel to carry out maintenance training, to improve the ability of maintenance personnel level and to meet the needs of equipment maintenance support has a very important significance.

Maintenance training simulation system of semi-physical simulation, physical simulation, virtual simulation and so on are commonly used, and the semi physical simulation maintenance system has the advantages of low cost and high efficiency, and has been widely used [1][2][3][4][5][6][7]. By a prototype of the equipment hydraulic system, by analysis on failure and repair processes in the difficult of the use of the actual operation of hydraulic system which often occurre, with Siemens S7-200 PLC and Kingview software as the main development tool, semi physical simulation maintenance training system with the function of the quick setup, fault simulation maintenance with the real-time monitoring and fault and troubleshooting of automatic detection and others is designed[8][9][10]. The main contents include the design of the system structure, the design of PLC control system, the software design based on kingview.

\section{System overall structure design}

\subsection{Structure of System}

The system consists of two layers, namely, the PLC field control layer and the upper computer monitoring layer. Field control uses Siemens S7-200 series PLC as the core of the control system to realise the function of the human-computer interaction and the data acquisition, including the operation panel of the hydraulic system, hydraulic system fault setting panel and maintenance of hydraulic system training panel. The operation signal of the panel in fact is collected and converted into digital signals by Siemens S7-200 programmable logic controller to transmit to the Kingview software. PC monitoring layer with Kingview software Kingview provides a realistic man-machine interface, which is convenient to display during normal operation and setting. The communication between PC and PLC is realized by OPC technology, PLC provide data service to the host computer 
by serial communication, through the operation in each panel, corresponding data is collected to the Kingview corresponding variables, and is unifiedly managed by the Kingview and the corresponding animation demonstration effect. Is produced The overall structure design is shown in figure1

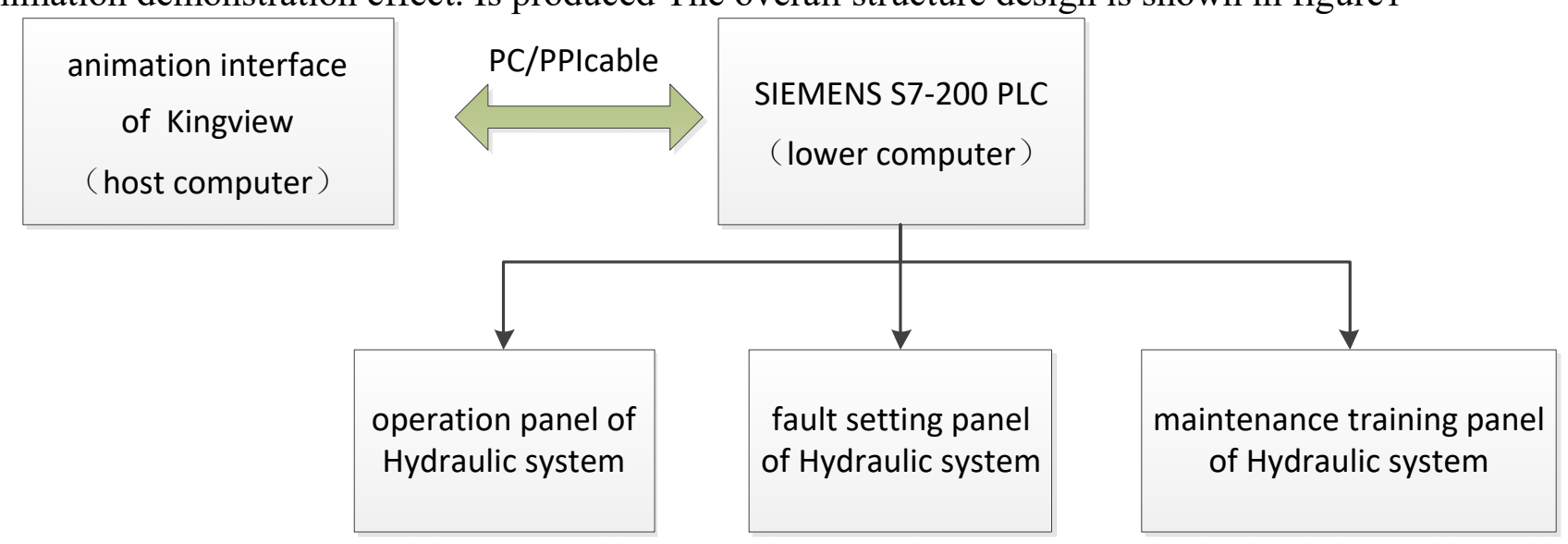

\subsection{Main function}

Fig. 1 Structure of system overall design

Main function:

(1) The operation panel of the hydraulic system is designed according to the hydraulic and electric operation panel, including all the hydraulic components of the control button, the normal operation process can be emulated by the panel, and the corresponding working principle is displayed in Kingview software, also once the corresponding operation does not conform to the normal process of logic, the appropriate warning and prompt disappear;

(2) Random set and directional set of common faults of hydraulic system are realized through the fault set panel, and failures in Kingview software, including reading digital tube display, change of hydraulic circuit flow effect, fault phenomenon of the implementation of components corresponding and alarm interface is presented;

(3) Set fault is repaired by the maintenance training panel, and the hydraulic system is restored to the normal working condition when the maintenance operation is correct.

\section{PLC system design of maintenance training system}

PLC system design of maintenance training system is mainly based on the relevant operation and function of the set of the hydraulic system operation panel, the hydraulic system fault setting panel and the hydraulic system maintenance training panel [11][12]. The communication between CPU and PLC is realized by STEP1-Micro/WIN32 programming software.

\subsection{Maintenance training system panel design}

Hydraulic system operation panel is simulated according to a actual operation panel, the operation panel for hydraulic and electric operation panel, leveling vertical control machine, flow control device and a pressure control device, leveling vertical power controller, parameter acquisition display panel in place signal display panel box, horizontal and vertical up vertical angle testing instrument panel are included. Panels are used to control the weapon equipment electromagnetic valve of hydraulic system YA1- YA28 and detect in place switch signal, pressure relay signal and the oil filter signal of hydraulic system and are showed through the indicator light, the signal is produced through the corresponding variables in the S7-200 PLC output to the Kingview software. According to the content of the panel, the operation panel is mainly controlled by the switch quantity. Operation panel of the PLC programming according to the order of operation process to take control, leveling up vertical, for example, only the normal unlocking circuit, in order to achieve erection leveling, in fact, only the operation of electromagnetic reversing valve YA2, YA22, YA23, YA24, YA25, operating YA6 will realize leveling erecting effect and YA2, ya22, YA23, YA24, YA25 also exist before and 
after the logical sequence, unlocking pump pressure start button the unlocking loop control button YA2, YA22, YA23, YA24, YA25 start normally.

Hydraulic system fault setting panel include the fault phenomenon involving the electromagnetic valve, hydraulic pump control component and actuator failure, the common fault phenomena is seted by settingthe cause of a fault,the fault reason is a hydraulic component fault working medium fault, the fault phenomenon is observed equipment failure, such as "unlock circuit pressure less than $20 \mathrm{MPa}$ ", the unlocking pressure is displayed by a pressure gauge , and fault reason is probably the temperature rise; electromagnetic reversing valve YA2, ya22, YA23, YA24, YA25 at least a leaked or not completely reset; the volumetric efficiency of the pump is decreased. When the fault reason is set, the fault phenomenon will prompt on the configuration interface and produce the corresponding animation.

Fault maintenance training panel is corresponding with fault setting panel, through the fault tree analysis on the failure phenomenon and maintenance on the cause of the fault, if the maintenance operation right is generated, hydraulic system reverted to normal animation and give tips, troubleshooting is controlled by switch quantity. Taking the fault setting and maintenance of YA2 solenoid valve as an example, the PLC control flow chart is shown in Figure 2.

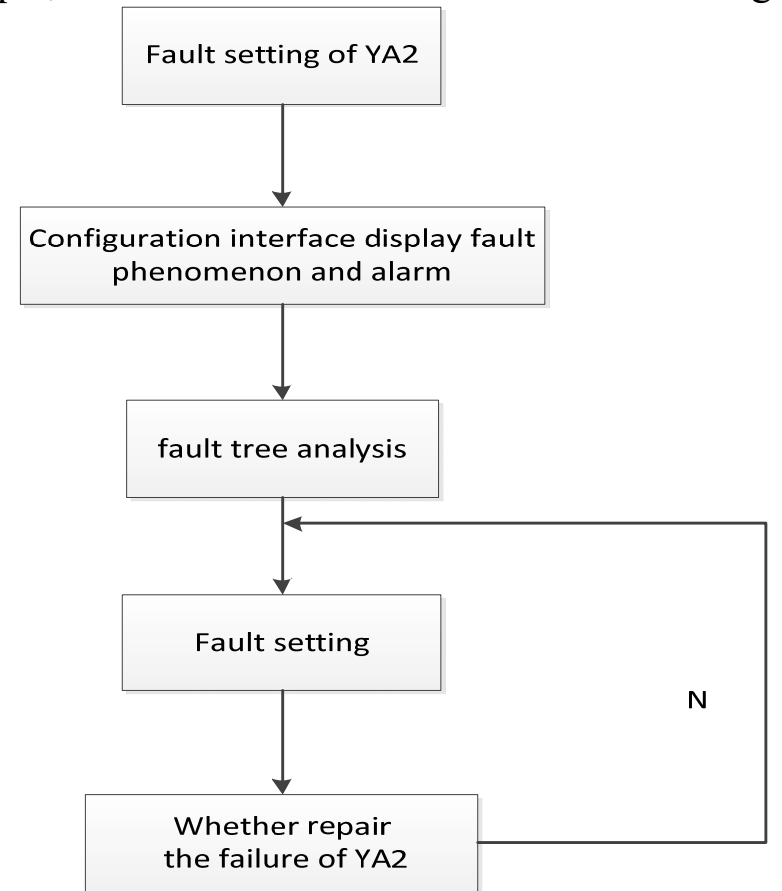

Figure 2 YA2 fault setting and maintenance PLC control flow chart

\subsection{PLC programming and $\mathrm{I} / \mathrm{O}$ distribution design}

Combined with the operation of YA2, YA 22, YA23, YA24, YA25 button and set faults and maintenance of content and distribution of I/O the PLC programming is showed in Figure 3, the fault setting and maintenance button of electromagnetic reversing valve YA2 is corresponding to the same input variables I4.1, belonging to the normally closed contact, the relationship between I1.1 and I4.1 is the logic and, relationship of YA22, YA23, YA24, YA25 belongs to logic or relations, with YA2 is logic and relation.YA22, YA23, YA24, YA25 failure setting and maintenance is same as button and YA2 . 


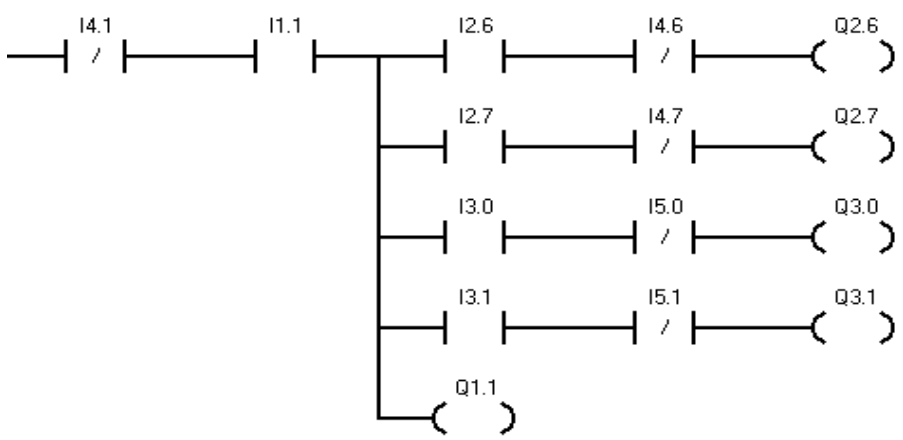

Figure 3 YA2, YA22, YA23, YA24, YA25PLC programming

Due to the complexity of an equipment of hydraulic structure and the implementation of components and control components with many kinds and large quantity, the system I/O distribution such as table 1 shows is only list, the register address VB250, VB251, VB252, VB253 respectively corresponding software Kingview variables accessed address.

Table 1 system part I/O distribution diagram

\begin{tabular}{|c|c|c|c|c|c|}
\hline Num & Input & Switch & Variable & Remark & Register \\
\hline 1 & I1. 0 & YA1 & YA1Button & Press for 1 & \multirow{8}{*}{ VB250 } \\
\hline 2 & I1. 1 & YA2 & YA2Button & Press for 1 & \\
\hline 3 & I1. 2 & YA3 & YA3Button & Press for 1 & \\
\hline 4 & I1. 3 & YA26 & YA26Button & Press for 1 & \\
\hline 5 & I1. 4 & YA19 & YA19Button & Press for 1 & \\
\hline 6 & I1. 5 & YA6 & YA6Button & Press for 1 & \\
\hline 7 & I1. 6 & YA8 & YA8Button & Press for 1 & \\
\hline 8 & I1. 7 & synchronization & $\begin{array}{c}\text { synchronization } \\
\text { مon Buttan }\end{array}$ & Press for 1 & \\
\hline 9 & I2. 0 & YA10 & YA10Button & Press for 1 & \multirow{8}{*}{ VB251 } \\
\hline 10 & I2. 1 & YA12 & YA12Button & Press for 1 & \\
\hline 11 & $\mathrm{I} 2.2$ & YA15 & YA15Button & Press for 1 & \\
\hline 12 & I2. 3 & YA20 & YA20Button & Press for 1 & \\
\hline 13 & $\mathrm{I} 2.4$ & YA16 & YA16Button & Press for 1 & \\
\hline 14 & I2. 5 & YA27 & YA27Button & Press for 1 & \\
\hline 15 & I2. 6 & YA22 & YA22Button & Press for 1 & \\
\hline 16 & I2. 7 & YA23 & YA23Button & Press for 1 & \\
\hline 17 & I3. 0 & YA24 & YA24Button & Press for 1 & \multirow{8}{*}{ VB252 } \\
\hline 18 & I3. 1 & YA25 & YA25Button & Press for 1 & \\
\hline 19 & I3. 2 & YA18 & YA18Button & Press for 1 & \\
\hline 20 & I3. 3 & YA7 & YA7Button & Press for 1 & \\
\hline 21 & I3. 4 & YA9 & YA9Button & Press for 1 & \\
\hline 22 & I3. 5 & synchronization & $\begin{array}{l}\text { synchronization } \\
\text { Withdraw Button }\end{array}$ & Press for 1 & \\
\hline 23 & I3. 6 & YA11 & YA11Button & Press for 1 & \\
\hline 24 & I3. 7 & YA13 & YA13Button & Press for 1 & \\
\hline 25 & I4. 0 & YA14 & YA14Button & Press for 1 & VB25333 \\
\hline
\end{tabular}

\section{Establishment of configuration project of maintenance training system}

Kingview software mainly includes three parts,Project Manager, project operation system and picture browser. The main functions of Kingview project manager include: construction engineering, project engineering, delete, search and modify the project properties. Engineering browser manages graphic screen, alarm, command language and other engineering elements centralizedly, the user is required to set the sub options for each project. In the operating system, screen, special, debugging, navigation menu is selected, operation interface of a project obtain data communication from the collection devices, and the dynamic picture is displayed according to animation design of project browser to achieve interactive operation and control equipment. Engineering establishment mainly includes the creation of the configuration screen, the definition of IO equipment, the construction of 
the database, the establishment of animation connection, operation and debugging and other major steps [13].

Due to the complexity of the hydraulic system, hydraulic system lock circuit is simplified, and the "unlocking circuit configuration screen" is established, simplified configuration screen is shown in Figure 4, black oil represents not work or failure state. Define YA2, YA22, YA23, YA24, YA25, as well as the animation connection of oil line object. According to the definition of the hydraulic system working principle , the electromagnetic reversing valve object animation for horizontal or vertical movement and oil object animation definition flow effect, the flow condition is electromagnetic valve action logic, in the pipeline attribute under the different working conditions define different colors of the circuit

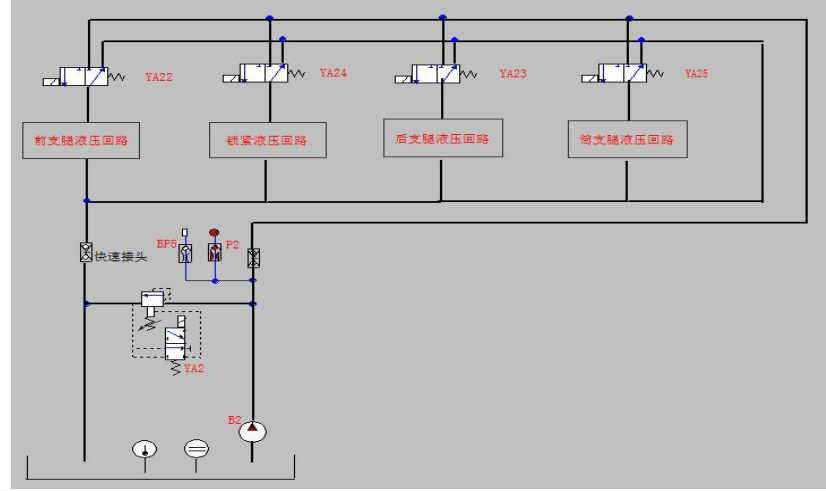

Figure 4 "unlock circuit" configuration screen

The normal working state as shown in Figure 5, YA2, YA22, YA23, YA24, YA25 all change, the corresponding circuit changes in color, white oil on behalf of the fuel tank, green oil on behalf of the working state when the lock oil normal.

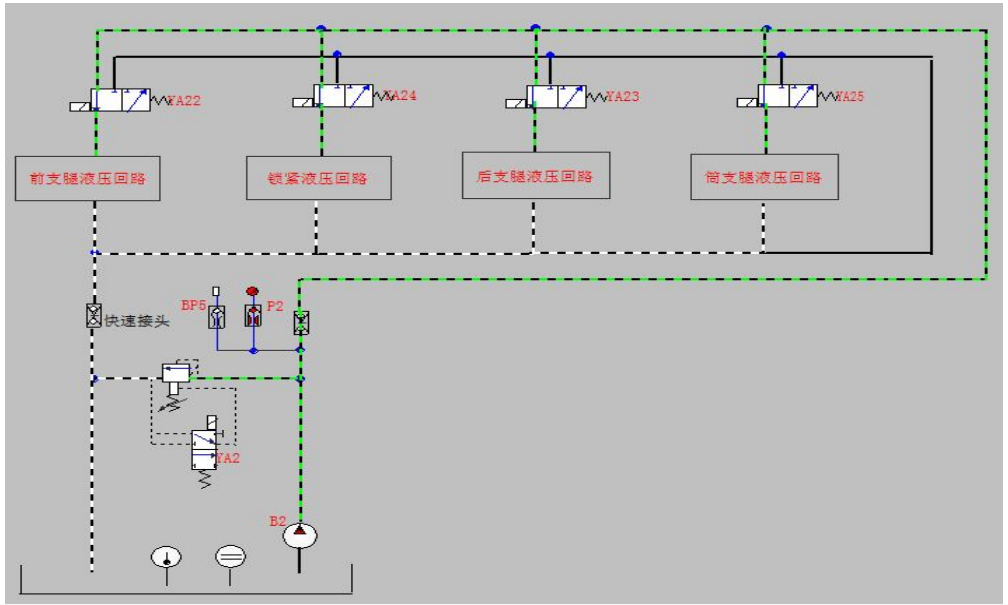

Figure 5 "unlock circuit" working status configuration screen

To unlock the oil pressure is less than $20 \mathrm{MPa}$ fault setting, for example, by fault tree analysis, the reason may is the system time is too long, the temperature; electromagnetic reversing valve YA2, ya22, YA23, YA24, YA25 at least have a leaked or not completely reset; the volumetric efficiency of the pump is decreased. To establish a "lock oil pressure less than 20MPa fault" screen fault, using showpictrue function to achieve fault screen and normal working status screen switching, define the temperature fault, YA2, ya22, YA23, YA24, YA25 fault, the volumetric efficiency of the pump down fault animation connection, when the button is pressed, fault screen as shown in Figure 6 . At this time due to the failure of YA2 lead to unlock oil pressure can not be reached 20MPa. Only when the YA2 fault button and the YA2 maintenance button are pressed at the same time, the normal working state can be returned to the working interface. 


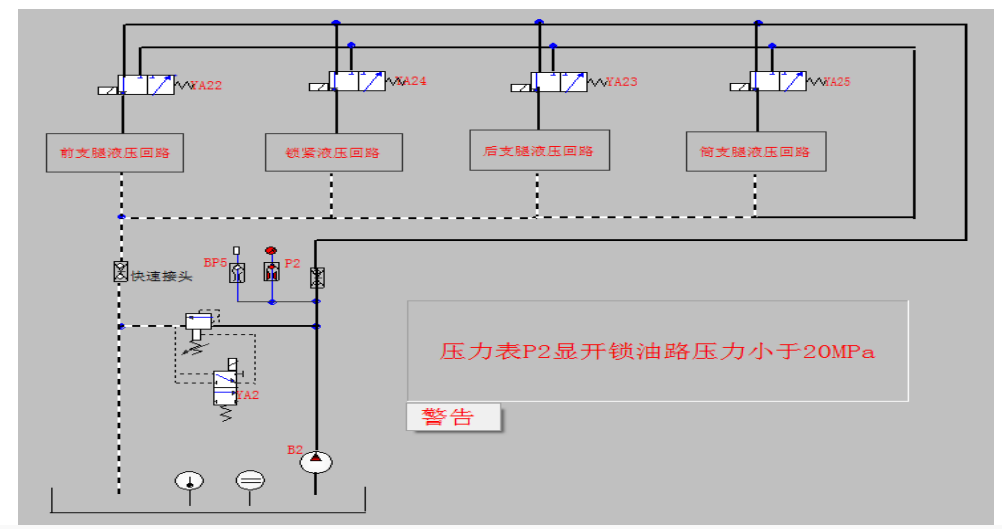

Figure 6 "unlock circuit" fault configuration screen

\section{Communication system}

Communication between PLC and Kingview is actually Kingview by logical device name of management on the management of the actual equipment, as shown in Figure 7, the specific $\mathrm{I} / \mathrm{O}$ devices and the logical device name is one-to-one, there is an I / $\mathrm{O}$ devices should be assigned a unique logical device name, especially equipment model exactly the same multiple I / O devices, also to specify different logical device name.

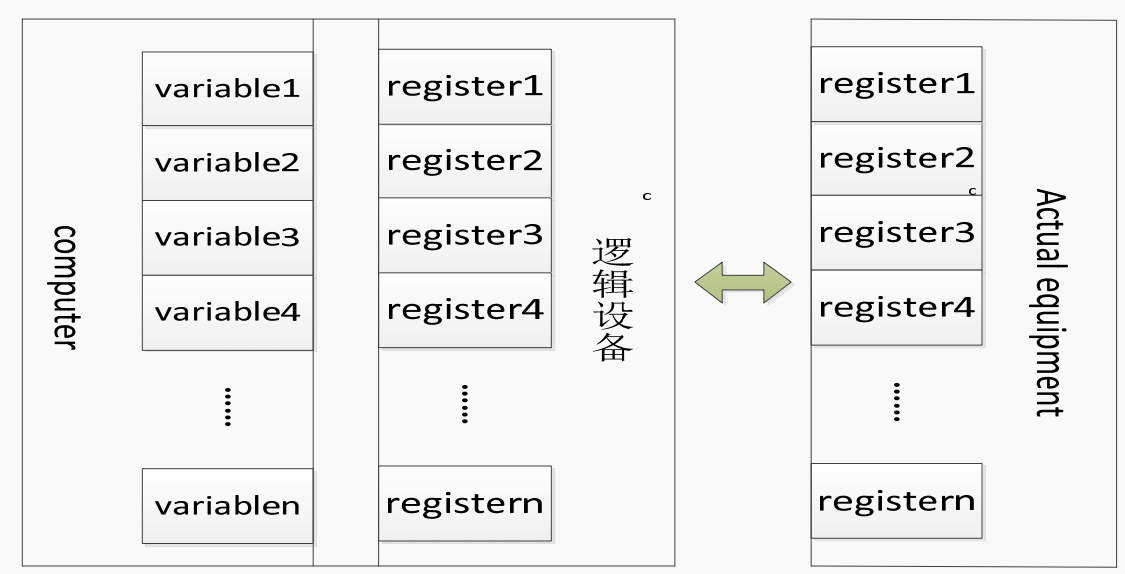

Figure 7 Schematic diagram of PLC and Kingview Communications

Supported by the Kingview hardware equipment comprises a programmable controller (PLC), intelligent module, a card, intelligent instrument, frequency converter, KingView to support the several means of communication: serial port communication, data acquisition board, DDE communication, man-machine interface card, network module, OPC. CPU through the PC/PPI cable and computer programming software STEP1-Micro/WIN32 communications, select the serial communication can meet the communication requirements between hardware and software.

After the completion of the definition of serial port equipment, to ya1 button in the variables defined in the configuration database, for example, as shown in Figure 8, type variables as discrete type that only 1 and 0 states, in the device configuration wizard choose Siemens S7-200 series (PPI), connecting device is automatically updated to Siemens PLC, according to the distribution of I / O, register definition for VB250.0 and data type of bit. The definition is completed, which can realize the communication between PLC and kingview. 


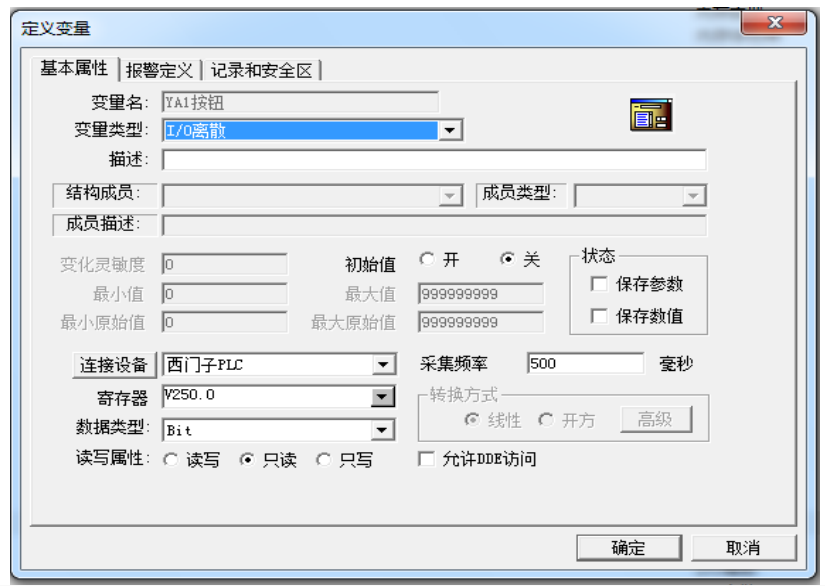

Figure 8 defines the YA1 button variable

\section{Summary}

Through the combination of PLC and configuration technology, using the PLC operation panel of the hydraulic system, fault setting panel and maintenance panel simulation and using KingView,hydraulic system working principle and fault principle of animation are displayed. Through the semi physical simulation,the functions of real-time display of oil flow, electromagnetic valve, hydraulic cylinder action has been realised, the working principle and faultare simulated, so that it makes the trainees have overall understanding and knowledge of working principle and fault principle of the hydraulic system, which greatly improves the efficiency of a hydraulic system equipment maintenance training, but also greatly reduces the cost of training and has some reference to other related training system.

\section{References}

[1] Huijun $\mathrm{Wu}$, Pingli Zhang,XiLiu et al. Application of PLC and Kingview in manipulator control system [J]. Electronic technology and software engineering, 2014 (13): 108-108.

[2] lili, et al. Automatic measurement system of gas flow meter based on PLC and configuration software [D]. University of Electronic Science and technology, 2013.

[3] Hangzhou Chen, et al. Tunnel ventilation control system based on PLC and Kingview [D]. Xiamen University, 2014.

[4] Wenbo Guan, Yize Sun, Zhuo Meng,et al. PLC and Kingview control system based on fiber modification [J]. Chemical automation and instrumentation, 2013, 40(12): 1466-1469.

[5] Yan B, Bei S, Zhiyi Z. Application of the S7-200PLC and KINGVIEW6.53to the constant pressure water supply system with variable frequency[J]. 2015.

[6] Wei Z, Xiaowen W. Research on hot-pressing machine control system based on PLC and Kingview[C].Control and Decision Conference (CCDC), 2015 27th Chinese. IEEE, 2015: 5644-5648.

[7] Biao Li, et al. Design and implementation of the system of liquid level control based on PLC and Kingview [J]. Journal of hunan university of science and engineering , 2013, 34(8): 24-27.

[8] Gang Yao, Wemyong Guo, Suhua Wang. The design of hydraulic steering gear maintenance training platform based on PLC and Kingview [J]. Hydraulic pneumatic and sealing , 2013 (10): 28-31.

[9] Baolin Wang. Design and application of PLC simulation teaching system of secondary vocational school based on configuration software [D]. Hunan Normal University , 2013.

[10] Chunfeng Zhao, Guomin Chen, Xiaolun Fan, et al . Research and development of electrical practice teaching device based on PLC [J]. Laboratory research and exploration

, 2013 (1): 136-139. 
[11]Jinling Fan. Design of pneumatic manipulator control system based on PLC [J]. Hydraulic and pneumatic, 2010, 2010(7): 36-38.

[12]Fengcai Huo, Weijian Ren, Chaohai Kang, et al. The design of the boiler monitoring system based on Kingview and PLC [J]. Chemical automation and instrumentation, 2013, 40(9): 1103-1105. [13] Hong Wang, Zicheng Wang, Guangzhao Cui. Research on control and Simulation of PLC elevator based on configuration software [J]. Manufacturing automation , 2013, 35(2): 109-112. 